\title{
Medios de control para un sistema de gestión de la innovación en las empresas manufactureras: caso agroindustria
}

\author{
Manuel Fernando Montoya Ramírez \\ Universidad de Lima. Lima, Perú \\ Correo electrónico: mmontora@ulima.edu.pe
}

Recibido: 27 de abril de 2015 / Aprobado: 28 de agosto de 2015

\begin{abstract}
Resumen: El presente artículo constituye el primer avance de una investigación que pretende proponer una metodología de diagnóstico sobre un sistema integrado de gestión de la innovación (SIGI), mediante una investigación cuantitativa y cualitativa en las empresas manufactureras del país. Se trata de definir los medios de control, luego sus indicadores, considerando la gestión del conocimiento, la gestión de la creatividad, la gestión tecnológica, la innovación de productos y la innovación del proceso como medios de control, a partir de las investigaciones realizadas a la fecha, y la aplicación de un cuestionario traducido y adaptado de uno validado, dirigido a las empresas agroindustriales del país como modelo, para luego ser replicado en otros sectores.
\end{abstract}

Palabras clave: gestión de la innovación / gestión del conocimiento / gestión de la creatividad / gestión de la tecnología / innovación de productos / innovación de procesos

\section{Control Means for a System of Innovation Management in Manufacturing Enterprises: Case Agribusiness}

AвSTRACт. This article constitutes the first step in research that aims to propose a methodology of diagnosis of an integrated management of innovation (SIGI), through quantitative and qualitative research in manufacturing companies in the country system. Define control means, then your indicators, considering knowledge management, creativity management, technology management, product innovation and process innovation as a means of control, from the research conducted to date, and applying a questionnaire translated and adapted from one validated, aimed at agribusiness companies in the country as a model, and then be replicated in other sectors.

Key words: innovation management / knowledge management / creativity management / technology management / innovation product $/$ process innovation 


\section{INTRODUCCIÓN}

Las innovaciones en productos y en procesos han jugado un papel importante en nuestra sociedad, contribuyendo al desarrollo de la humanidad y a mejorar la calidad de vida, sobre todo gracias a los cambios tecnológicos; sin embargo, luego de muchas discusiones acerca de cómo definir la innovación pura, se ha podido establecer que es una idea nueva cuya aplicación es exitosa, y constituye una aportación significativa no solo para la empresa sino también para los clientes, en la medida en que es el camino más apropiado para lograr una mayor productividad y mejora sustancial del crecimiento económico.

En nuestro país existen esfuerzos significativos en el sector manufacturero por realizar innovaciones de forma incremental para lograr una mejora continua, pero se observa que no se encuentra la innovación estructurada como un sistema que pueda ser controlado en forma permanente, por lo que es necesario contar con medios que permitan medir la situación, el avance y los resultados de una gestión de la innovación, para tomar decisiones de manera oportuna y adecuada. Los trabajos de investigación acerca de la gestión de control de la innovación siguen siendo escasos (Poskela y Martinsuo, 2009).

Sin embargo, existen estudios que buscan establecer formas de medir la gestión de la innovación, como el de Hagedoorn y Cloodt (2003), que propone diferentes indicadores sobre el desempeño de innovación, pero vinculándolos con la inscripción de patentes. A su vez, ArévaloTomé, Urgal y Quintas (2011) realizan una revisión bibliográfica de los indicadores de desempeño innovador; por su parte, Prajogo y Ahmed (2006) examinan el modo de integrar los aspectos humanos y tecnológicos a la gestión de la innovación, mediante los factores que la estimulen, y cómo relacionarlos con la capacidad para determinar el rendimiento de la innovación.

Sobre la innovación de productos la investigación indica que el funcionamiento y el rendimiento del producto son dos aspectos diferentes, si se toma en cuenta el sesgo de los métodos comunes y que el rendimiento operativo es una variable formativa y el rendimiento del producto indica una variable reflexiva (Molina-Castillo y Munuera-Alemán, 2009). Además, para medir el rendimiento de la innovación crean un indicador que resume el impacto de la innovación en las actividades de los productos, los procesos y la sostenibilidad, y concluye que el conocimiento no solo tiene un efecto positivo directo en el rendimiento de la innovación sino 
también un efecto indirecto, mediante la mejora de la empresa en su capacidad para innovar y arroja luz sobre cómo las empresas pueden aprovechar sus recursos de conocimiento para obtener una ventaja competitiva basada en la innovación (Arévalo-Tomé, Urgal y Quintas, 2011).

En todos los casos estos medios de control se presentan de forma aislada y no como un sistema modelado para obtener un control permanente de la innovación de la empresa, sino que toman en cuenta el rendimiento de la innovación en un momento dado pero no en forma continua, y es justamente esto lo que se plantea; es decir, encontrar un grupo de medios que controlen un sistema de gestión en -este caso la innovación- en forma permanente, y que ese control se constituya en un medio para que las empresas eleven su rendimiento por el camino de la innovación, al contar con indicadores que midan la gestión de la innovación, y determinen su situación señalando los aspectos más relevantes y que generen oportunidades de mejora.

\section{MATERIALES Y MÉTODOS}

Para determinar los medios que controlen el sistema de gestión de la innovación en las empresas, se estableció la necesidad de contar con la opinión de los trabajadores independientemente de sus cargos, para no sesgar el resultado solo con el parecer de la alta gerencia; además, se segmentó el sector manufacturero utilizando únicamente el agroindustrial, en razón de que ese sector mantiene una ventaja competitiva en disponibilidad de mano de obra, costos de energía, tecnología de vanguardia, como señala Alejandro Fuentes, gerente general de Agrícola Don Ricardo, en la entrevista concedida al diario Gestión (Bardales, 14 de noviembre de 2014), así como el crecimiento en las exportaciones no tradicionales y la generación de puestos de trabajo en el país en los últimos años.

Se seleccionaron 120 empresas del sector agroindustrial de nuestro país, entre grandes , medianas y pequeñas empresas. Luego se estudiaron las fuentes secundarias, como las revistas científicas en las que se exponen las formas como se han establecido los controles en la innovación, y por último se seleccionó y adaptó un cuestionario para validar y contrastar los resultados.

El cuestionario utilizado fue el resultado de la traducción y adaptación de uno que se utilizó en Australia en el año 2001, con una encuesta 
a mil directivos que tenían conocimiento de calidad e innovación de varios sectores industriales. La muestra fue seleccionada al azar por la Organización Australiana para la Calidad (AOQ), cuya base de datos también abarca varios sectores industriales. El enfoque de dicho estudio se limitó a un sitio (o planta) por organización (Prajogo y Ahmed, 2006), por lo que ha sido modificado para adecuarlo a nuestra realidad, considerando el objetivo de la presente investigación, puesto que nos dirigimos al sector manufacturero agroindustrial. Además, el modelo de escala que se utilizará es la de Likert (1932), con puntuaciones del 1 al 5, de totalmente en desacuerdo a totalmente de acuerdo.

El objetivo es establecer si son correctos los medios de control para un sistema integrado de gestión de la innovación (SIGI) en el sector manufacturero que se proponen en la presente investigación, a partir de la revisión de la literatura detallada en la introducción, como se muestra en la figura 1, y cuyo cuestionario está compuesto por secciones. En la primera se plantean preguntas demográficas, como género, edad, formación y antigüedad en la empresa. Luego están los bloques de preguntas relacionadas con los siguientes medios de control: la gestión del conocimiento (GC), la gestión de la creatividad (GCR), la gestión tecnológica (GT), la innovación de productos (INP) y la innovación de procesos (IPR), para probar si estos pueden mediar la gestión de la innovación. Por último, las respuestas de los cuestionarios fueron ingresados al software estadístico SPSS para tabular y analizar los resultados, realizándose un análisis factorial.

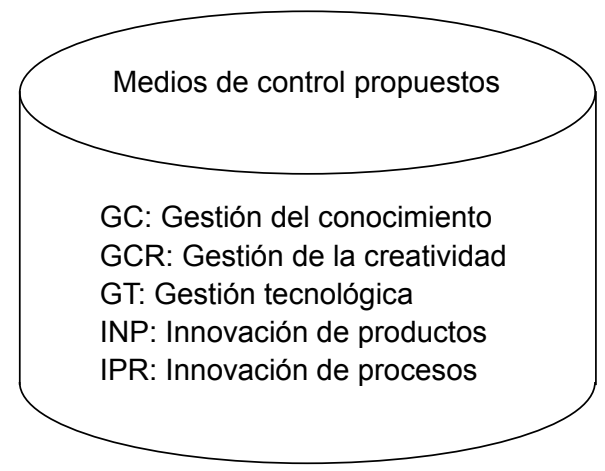

Figura 1. Sistema integrado de gestión de la innovación (SIGI)

Elaboración propia 


\section{RESULTADOS}

Se contactaron 120 empresas agroindustriales del país, de las cuales 62 respondieron el cuestionario de nuestra investigación (30 \% de empresas grandes y $70 \%$ de empresas medianas y pequeñas); y con la finalidad de conocer las respuestas de los trabajadores, independientemente de su cargo en las empresas, se consideraron aspectos como el nivel académico, el género y los años en la empresa. En la tabla 1 se muestran los resultados de su nivel académico: el 43,5\% son profesionales titulados, el $35 \%$ tiene el grado de magíster, el 9,7\% son bachilleres, el 6,5\% cuentan con nivel técnico, y el 4,8\% son doctores.

\begin{tabular}{lcc}
$\begin{array}{l}\text { Tabla } 1 \\
\text { Nivel académico }\end{array}$ & $\begin{array}{c}\text { N. }{ }^{\circ} \text { de } \\
\text { trabajadores }\end{array}$ & $\%$ \\
\hline Técnico & 4 & 6,5 \\
Bachiller & 6 & 9,7 \\
Profesional & 27 & 43,5 \\
Magíster & 22 & 35,0 \\
Doctor & 3 & 4,8 \\
\hline Total & $\mathbf{6 2}$ & $\mathbf{9 9 , 5}$ \\
\hline
\end{tabular}

Elaboración propia

En cuanto a la antigüedad en la empresa, para efectos de simplificar el análisis se han establecido rangos en años y se obtuvo el siguiente resultado: hasta 5 años $48,4 \%$, entre 6 y 10 años $30,6 \%$, entre 11 y 15 años $12,9 \%$, y entre 16 y 35 años $8,1 \%$, por lo tanto, más del $30 \%$ tiene una antigüedad de más de 5 años en la empresa, con lo que se concluye que existe un conocimiento de la empresa y su entorno.

Otra información obtenida de las encuestas es la edad de los entrevistados, en la que, como en el caso anterior, se han establecido rangos para simplificar el análisis: el 35,5\% tiene entre 29 y 35 años; el $33,9 \%$ entre 36 y 50 años, el 17,7 \% entre 51 y 70 años, y el 12,9 \% entre 22 y 28 años; es decir, más del $80 \%$ de los encuestados tiene más de 29 años de edad, por lo que cuentan con la madurez laboral suficiente para responder la encuesta. 
Tabla 2

Antigüedad en la empresa

\begin{tabular}{lcc}
\hline & $\begin{array}{c}\mathbf{N}^{\circ} \text { de } \\
\text { Trabajadores }\end{array}$ & $\%$ \\
\hline Hasta 5 años & 30 & 48,4 \\
De 6 a 10 años & 19 & 30,6 \\
De 11 a 15 años & 8 & 12,9 \\
De 16 a 35 años & 5 & 8,1 \\
\hline Total & $\mathbf{6 2}$ & $\mathbf{1 0 0}$ \\
\hline
\end{tabular}

Elaboración propia

Tabla 3

Edad

\begin{tabular}{lcc}
\hline & $\begin{array}{c}\mathbf{N}^{\circ} \text { de } \\
\text { trabajadores }\end{array}$ & $\%$ \\
\hline De 22 a 28 años & 8 & 12,9 \\
De 29 a 35 años & 22 & 35,5 \\
De 36 a 50 años & 21 & 33,9 \\
De 51 a 70 años & 11 & 17,7 \\
\hline Total & $\mathbf{6 2}$ & $\mathbf{1 0 0}$ \\
\hline
\end{tabular}

Elaboración propia

\subsection{Gestión del conocimiento (GC)}

En relación con los medios de control se ha obtenido la siguiente información en cuanto a la gestión del conocimiento (GC), definida como la capacidad que tienen las empresas de crear, utilizar y compartir conocimientos. El $23 \%$ está totalmente de acuerdo y el 39,5\% está de acuerdo, considerando las respuestas de las cuatro preguntas que conforman esta categoría, por lo tanto, más del $50 \%$ acepta este medio como válido o adecuado para medir la gestión de la innovación en la empresa.

Asimismo, se realizó la prueba KMO (medida Kaiser-Meyer-Olkin) de adecuación de muestreo, en el que se obtiene un valor de 0.778; el $\mathrm{KMO}$ entre 0.5 y 0.8 indica que la muestra global es aceptable para realizar el análisis factorial de los ítems del cuestionario respecto al constructo gestión del conocimiento (GC); además, los valores son sig- 
nificativos, ya que el coeficiente de correlación de la pregunta es mayor a su p-valor. Sobre el determinante de la matriz, como tiene un valor de 0.270 , es menor a 0,4 , indica que hay altas correlaciones entre las preguntas que se están analizando. La matriz factorial y los índices de correlación se muestran en la tabla 5.

\begin{tabular}{lcc}
$\begin{array}{l}\text { Tabla } 4 \\
\text { Gestión del conocimiento (GC) }\end{array}$ & \\
\hline & $\begin{array}{c}\text { N. }{ }^{\circ} \text { de respuesta } \\
\text { por escala }\end{array}$ & $\%$ \\
\hline 1 Totalmente en desacuerdo & 0 & 0 \\
2 & 21 & 8,5 \\
3 & 72 & 29,0 \\
4 & 98 & 39,5 \\
5 Totalmente de acuerdo & 57 & 23,0 \\
\hline Total & $\mathbf{2 4 8}$ & $\mathbf{1 0 0}$
\end{tabular}

Elaboración propia

Tabla 5

Matriz factorial

\begin{tabular}{cc}
\hline Ítem & $\begin{array}{c}\text { Índice de } \\
\text { correlación }\end{array}$ \\
\hline P1 & 0.682 \\
P2 & 0.804 \\
P3 & 0.849 \\
P4 & 0.833 \\
\hline
\end{tabular}

Elaboración propia

Todos los índices de correlación son mayores a 0.6 , por lo tanto los ítems utilizados describen el constructo (GC) establecido.

\subsection{Gestión de la creatividad (GCR)}

También se analizó la gestión de la creatividad (GCR), referida al nivel de motivación y desafío de los equipos de trabajo, así como los recursos 
destinados para la innovación. El cuestionario está compuesto por cuatro preguntas, y muestra el siguiente resultado: totalmente de acuerdo $16,5 \%$, de acuerdo $37,1 \%$; lo que indica que más del $50 \%$ lo considera como válido o adecuado para medir la gestión de la innovación, tal como se muestra en la tabla 6.

Tabla 6

Gestión de la creatividad (GCR)

\begin{tabular}{lcc}
\hline & $\begin{array}{c}\mathbf{N}^{\circ} \text { de respuestas } \\
\text { por escala }\end{array}$ & $\%$ \\
\hline 1 Totalmente en desacuerdo & 5 & 2,0 \\
2 & 32 & 12,9 \\
3 & 78 & 31,5 \\
4 & 92 & 37,1 \\
5 Totalmente de acuerdo & 41 & 16,5 \\
\hline Total & $\mathbf{2 4 8}$ & $\mathbf{1 0 0}$ \\
\hline
\end{tabular}

Elaboración propia

También se realizó la prueba KMO de adecuación de muestreo, en el que se obtiene un valor de 0.748 , el KMO entre 0.5 y 0.8 indica que la muestra global es aceptable para realizar el análisis factorial de los ítems del cuestionario respecto al constructo gestión de la creatividad (GCR); además, los valores son significativos, ya que el coeficiente de correlación de la pregunta es mayor a su p-valor. El determinante de la matriz de correlación es de 0.364 , como tiene un valor menor a 0,4 indica que hay altas correlaciones entre las preguntas que se están analizando. La matriz factorial y los índices de correlación se muestran en la tabla 7.

Tabla 7

Matriz factorial (GCR)

\begin{tabular}{cc}
\hline Ítem & Índice de correlación \\
\hline P1 & 0.759 \\
P2 & 0.776 \\
P3 & 0.657 \\
P4 & 0.851 \\
\hline
\end{tabular}

Elaboración propia 
Todos los índices de correlación son mayores a 0.6 , por lo tanto los ítems utilizados describen el constructo (GCR) establecido.

\subsection{Gestión de la tecnología (GT)}

El siguiente medio de control analizado es gestión de la tecnología (GT), definido como las innovaciones tecnológicas propuestas en la empresa, los profesionales dedicados a innovar tecnológicamente y el presupuesto destinado para investigación y desarrollo. Está compuesto por cuatro preguntas, y de la encuesta el 16,9 \% está totalmente de acuerdo, el 40,7 \% de acuerdo, por lo tanto más del $50 \%$ de los encuestados da como válido o adecuado para medir la gestión de la innovación, como se muestra en la tabla 8 .

Tabla 8

Gestión de la tecnología (GT)

\begin{tabular}{lcc}
\hline & $\begin{array}{c}\mathbf{N}^{\circ} \text { de respuestas } \\
\text { por escala }\end{array}$ & $\%$ \\
\hline 1 Totalmente en desacuerdo & 2 & 0,8 \\
2 & 28 & 11,3 \\
3 & 75 & 30,2 \\
4 & 101 & 40,7 \\
5 Totalmente de acuerdo & 42 & 16,9 \\
\hline Total & $\mathbf{2 4 8}$ & $\mathbf{1 0 0}$ \\
\hline
\end{tabular}

Elaboración propia

Además, se realizó la prueba $\mathrm{KMO}$ de adecuación de muestreo, en el que se obtiene un valor de 0.776, el KMO entre 0.5 y 0.8 indica que la muestra global es aceptable para realizar el análisis factorial de los ítems del cuestionario respecto al constructo gestión de la tecnología (GT); además, los valores son significativos, ya que el coeficiente de correlación de la pregunta es mayor a su p-valor. El determinante de la matriz de correlación es de 0.236 , como tiene un valor menor a 0,4 indica que hay altas correlaciones entre las preguntas que se están analizando. La matriz factorial y los índices de correlación se muestran en la tabla 9 . 
Tabla 9

Matriz factorial (GT)

\begin{tabular}{cc}
\hline Ítem & Índice de correlación \\
\hline P1 & 0.759 \\
P2 & 0.776 \\
P3 & 0.657 \\
P4 & 0.851 \\
\hline
\end{tabular}

Elaboración propia

Todos los índices de correlación son mayores a 0.6 , por lo tanto los ítems utilizados describen el constructo (GT) establecido.

\subsection{Innovación de productos (INP)}

En cuanto a la innovación de productos (INP), es considerado como medio de control y definido como búsqueda de información del mercado, novedad percibida de los productos lanzados al mercado y solución de problemas existentes en el mercado. Consta de cinco preguntas, de las cuales se obtiene que el 10,9\% está totalmente de acuerdo y el 33,5\% está de acuerdo, por lo tanto, menos del $50 \%$ lo valida como medio de control para la gestión de la innovación; sin embargo, el 41,9 \% ni lo aprueba ni lo desaprueba; además, debemos considerar que el 12,9\% está en desacuerdo y solo el 0,8\% está totalmente en desacuerdo, por lo tanto este medio podría controlar la gestión de la innovación al final del proceso, no en la propia gestión, pero sí para medir el resultado, como se muestra en la tabla 10.

Tabla 10

Innovación de productos (INP)

\begin{tabular}{lcc}
\hline & $\begin{array}{c}\mathbf{N}^{\circ} \text { de respuesta } \\
\text { por escala }\end{array}$ & $\%$ \\
\hline 1 Totalmente en desacuerdo & 2 & 0,8 \\
2 & 32 & 12,9 \\
3 & 104 & 41,9 \\
4 & 83 & 33,5 \\
5 Totalmente de acuerdo & 27 & 10,9 \\
\hline Total & $\mathbf{2 4 8}$ & $\mathbf{1 0 0}$ \\
\hline
\end{tabular}

Elaboración propia 
Asimismo, se realizó la prueba KMO de adecuación de muestreo, en el que se obtiene un valor de 0.751 , el KMO entre 0.5 y 0.8 indica que la muestra global es aceptable para realizar el análisis factorial de los ítems del cuestionario respecto al constructo innovación de productos (INP); además, los valores son significativos, ya que el coeficiente de correlación de la pregunta es mayor a su p-valor. El determinante de la matriz de correlación da 0.333 , como tiene un valor menor a 0,4 indica que hay altas correlaciones entre las preguntas que se están analizando. La matriz factorial y los índices de correlación se muestran en la tabla 11.

Tabla 11

Matriz factorial (INP)

\begin{tabular}{cc}
\hline Ítem & Índice de correlación \\
\hline P1 & 0.855 \\
P2 & 0.639 \\
P3 & 0.770 \\
P4 & 0.806 \\
\hline
\end{tabular}

Elaboración propia

Todos los índices de correlación son mayores a 0.6 , por lo tanto los ítems utilizados describen el constructo (INP) establecido.

\subsection{Innovación en procesos (IPR)}

Por último, se analizó la innovación de procesos (IPR) como medio de control, definido como mejora en los procesos, aumento de la productividad y el tiempo establecido en el cuello de botella del proceso. Consta de cuatro preguntas, de las que se obtuvo que el $11,7 \%$ está totalmente de acuerdo y el 38,3 \% está de acuerdo, por lo tanto menos del $50 \%$ lo valida como medio de control de la gestión; sin embargo, el 37,9 \% ni lo aprueba ni desaprueba; además, debemos considerar que el 11,7 \% está en desacuerdo y solo el 0,4 \% está totalmente en desacuerdo, por lo tanto este medio también podría controlar la gestión de la innovación al final, como el resultado de todo el proceso, como se muestra en la tabla 12.

Luego se realizó la prueba KMO de adecuación de muestreo, en el que se obtiene un valor de 0.742 ; el KMO entre 0.5 y 0.8 indica que la muestra global es aceptable para realizar el análisis factorial de los ítems 
del cuestionario respecto al constructo innovación de productos (INP); además, los valores son significativos, ya que el coeficiente de correlación de la pregunta es mayor a su p-valor. El determinante de la matriz de correlación da 0.216 , como tiene un valor menor a 0,4 indica que hay altas correlaciones entre las preguntas que se están analizando. La matriz factorial y los índices de correlación se muestran en la tabla 13.

Tabla 12

Innovación de procesos (IPR)

\begin{tabular}{lcc}
\hline & $\begin{array}{c}\mathbf{N}^{\circ} \text { de respuesta } \\
\text { por escala }\end{array}$ & $\%$ \\
1 Totalmente en desacuerdo & 1 & 0,4 \\
2 & 29 & 11,7 \\
3 & 94 & 37,9 \\
4 & 95 & 38,3 \\
5 Totalmente de acuerdo & 29 & 11,7 \\
\hline Total & $\mathbf{2 4 8}$ & $\mathbf{1 0 0}$ \\
\hline Elaboración propia & &
\end{tabular}

Tabla 13

Matriz factorial (IPR)

\begin{tabular}{cc}
\hline Ítem & Índice de correlación \\
\hline P1 & 0.782 \\
P2 & 0.860 \\
P3 & 0.844 \\
P4 & 0.731 \\
\hline
\end{tabular}

Elaboración propia

Todos los índices de correlación son mayores a 0.6 , por lo tanto los ítems utilizados describen el constructo (IPR) establecido.

\subsection{Sistema integrado de gestión de la innovación (SIGI)}

Por último, en el análisis factorial se examinaron todas la relaciones de los medios de control propuestos en parejas, para establecer si existen intercorrelaciones entre las variables, es decir si existen o no influencias 
entre ellas mediante el determinante, que debe ser menor a 0.4 , y entre más bajo mayor intercorrelación, que nos permita, además, establecer una propuesta de sistema integrado de gestión de la innovación (SIGI), obteniendo los valores más bajos, como se muestran en la tabla 14 .

Tabla 14

Determinantes

\begin{tabular}{cc}
\hline $\begin{array}{c}\text { Relación entre } \\
\text { variables }\end{array}$ & Determinantes \\
\hline GC - GT & 0.038 \\
GC - GCR & 0.042 \\
GT - GCR & 0.048 \\
GCR - INP & 0.122 \\
GCR - IPR & 0.089 \\
INP - IPR & 0.109 \\
\hline
\end{tabular}

Elaboración propia

\section{DISCUSIÓN}

La gestión de la innovación se define como la invención y la aplicación de una práctica de gestión de procesos, estructura o técnica que es nueva en la empresa y genera beneficios (Birkinshaw, Hamel y Mol, 2008). Tras examinar la vasta literatura de innovación se llega a una definición clara del constructo orientación hacia la innovación, con el fin de ofrecer una conceptualización coherente para las investigaciones futuras (Siguaw, Simpson y Enz, 2006).

Al examinar el campo de la innovación se encontró un vacío en las investigaciones sobre la necesidad de analizar los factores que afectan la gestión de la innovación y el conocimiento en los trabajadores (Bhatnagar, 2014). En un estudio basado en Internet, con una muestra étnicamente diversa de 1337 directivos de 19 países (principalmente de Estados Unidos y Canadá), se obtuvo un hallazgo espectacular, consistente con otras investigaciones sobre habilidades directivas, el cual señala que la mejora de la creatividad en los negocios y la industria es más alta para las personas que habían tenido formación en gestión de la creatividad (GCR) y se correlacionaron positivamente con el número de horas de capacitación (Epsstein, Kaminaka, Phan y Uda, 2013). 
La integración del conocimiento se está convirtiendo en un reto fundamental para la gestión de la innovación. Si bien en la literatura recibida se reconoce la importancia de la interdisciplinariedad, poco se sabe sobre sus antecedentes teóricos y conceptuales, orientados a identificar los desafíos para la gestión de la innovación y formular implicaciones en las futuras investigaciones (Hacklin y Wallin, 2013). En la presente investigación, de acuerdo con los resultados obtenidos, la gestión del conocimiento $(\mathrm{GC})$ se constituye en un elemento para medir la gestión de la innovación, como un sistema estructurado y de forma continua en las empresas. Sin embargo, se muestra a la innovación como un resultado que no ha sido claramente definido desde una perspectiva del conocimiento (Quintane, Casselman, Sebastian y Nylund, 2011).

Los modelos de gestión innovadores consideran que los recursos humanos son una dimensión clave en el proceso de innovación y en su rendimiento. En particular, el conocimiento de las personas y su creatividad es fundamental para la innovación. Sin embargo, poco se ha estudiado acerca de qué tipo de prácticas de gestión de recursos humanos fomenta la mejora de los conocimientos y la creatividad, las actitudes y el comportamiento en el personal que va a conducir a los mejores resultados en materia de innovación. En concreto, se sostiene que existe una relación directa, así como un posible efecto moderador, de la orientación estratégica creativa hacia la innovación (PerdomoOrtiz, Gonzales-Benito y Galende, 2009). También Gonzales-Mieres, Santos-Vijande y López-Sánchez (2013) señalan en su investigación una perspectiva organizativa para abordar la cocreación, analizando cómo diversos tipos culturales de organización (la capacidad de innovación, la evaluación de los empleados de primera línea y los clientes como cocreadores) interactúan y contribuyen a la competitividad.

Se argumenta que la relación entre el clima y el equipo de la innovación será más fuerte para los equipos de investigación que los equipos de desarrollo, ya que los equipos de investigación tienen un mayor margen para la creación de ideas nuevas e innovadoras (Bain, Mann y Pirola-Merlo, 2001). También se ha desarrollado un marco para evaluar la innovación mediante la capacidad inventiva, los patrones de especialización tecnológica, la apertura hacia la innovación y el potencial económico de la tecnología. De esta manera, el marco propuesto sirve como una herramienta para evaluar el potencial de la colaboración creativa y anticipar los beneficios de esa colaboración, siempre tomando como base la tecnología (De Prato y Nepelski, 2013), por eso 
se establece la gestión de la creatividad (GCR) como un instrumento en las empresas para medir la gestión de la innovación y que ha sido confirmado en el resultado de nuestro cuestionario, de acuerdo al análisis factorial y al análisis estadístico presentado.

También se plantea como medida de control de la gestión de la innovación la gestión de la tecnología (GT), que fue considerada como aceptable, por los resultados de la encuesta mostrados anteriormente. Sin embargo, el papel de la gestión de la innovación, al permitir la innovación de procesos tecnológicos en un contexto interorganizacional, sigue siendo en gran parte inexplorado (Hollen, Van den Bosch y Volverda, 2012).

La capacidad de integrar la innovación con la transferencia tecnológica y organizativa es muy importante para el control de la gestión de la innovación, más aún si se hace con un enfoque en sus indicadores, lográndose de esa manera una nueva comprensión de la gestión de la innovación (Pohlmann, Gebhardt y Etzkowitz, 2005). Las investigaciones sobre la innovación en las organizaciones ha seguido principalmente un enfoque tecnológico, volcando todos sus esfuerzos de innovación a través de actividades de I + D (investigación y desarrollo). Las teorías de la innovación en su mayoría están conformadas por los estudios de las innovaciones tecnológicas en el sector manufacturero, pero se aplican en todos los contextos, como en modificar los procesos de gestión de la organización y los sistemas administrativos, motivar y recompensar a sus miembros y permitir su adaptación al cambio organizativo (Vangelis, 2002), así como aumentar la comprensión de la innovación empresarial a partir de las teorías de la innovación derivada de los estudios de los cambios tecnológicos y por lo tanto ayudar a extender sus avances y la investigación sobre el proceso de innovación y sus resultados (Damanpour y Aravind, 2012).

Los medios de control de innovación de productos (INP) y de procesos (IPR), si bien no han sido rechazados por los encuestados, estos solo los aceptan medianamente, sin embargo se van a utilizar para controlar la gestión de la innovación en su resultado y no en la gestión propiamente dicha. Cabe recalcar que en el proceso de gestión y la innovación no existe un método significativamente excepcional para medir y monitorear el nivel de innovación en los procesos.

Para conocer con precisión el estado de la innovación de los procesos de fabricación, se utilizan indicadores que solamente miden el producto final, mas no la gestión en cada una de las etapas del proceso 
(Ayhan, Oztemel, Aydin y Yue, 2013). La innovación de procesos puede permitir elevar la eficiencia y la eficacia, que son indicadores claves de la ventaja competitiva a largo plazo de las empresas manufactureras. Sin embargo, la literatura sobre gestión de la innovación de procesos es amplia y fragmentada, y no ha sido revisada aún en forma sistemática en la literatura académica (Frishammar, Kurkkio, Abrahamsson y Lichtenthaler, 2012).

\section{CONCLUSIONES}

El éxito de las estrategias de cambio o el establecimiento de la innovación de sistemas es indicado o monitoreado por las innovaciones científicas y tecnológicas, y el número de nuevos productos y patentes, pero la gestión de la innovación como un sistema estructurado se encuentra en un segundo plano; por este motivo se plantea primero controlar el sistema, para luego obtener esos resultados deseados, tanto en productos como en procesos, para lo cual se propone como medio de control que la gestión del conocimiento (GC), la gestión de la creatividad (GCR) y la gestión de la tecnología (GT) son medios adecuados para controlar la gestión de la innovación como un sistema; asimismo, la innovación en productos (INP) y la innovación en procesos (IPR) son medios adecuados para evaluar los resultados de una gestión de la innovación en las empresas, como se muestra en la figura 2.

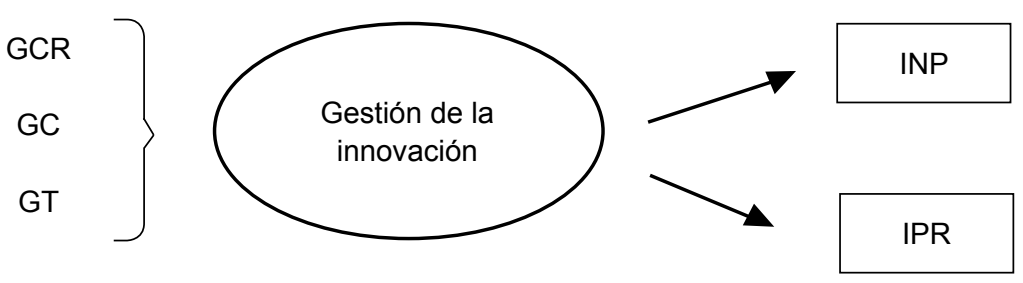

Figura 2. Medios de control de la gestión de la innovación

Elaboración propia

Además, de acuerdo al análisis factorial, mediante los determinantes para cada medio de control, que comprueba la relación de los ítems del cuestionario con cada medio de control y los determinantes mostrados en la tabla 14, se puede plantear un sistema integrado de gestión de la innovación (SIGI), como se muestra en la figura 3, que permitan a las empresas utilizar una forma de controlar, así como también un 
sistema estructurado, que demuestre la relación entre los diferentes medios de control para obtener una gestión de la innovación permanente en las empresas de manufactura.

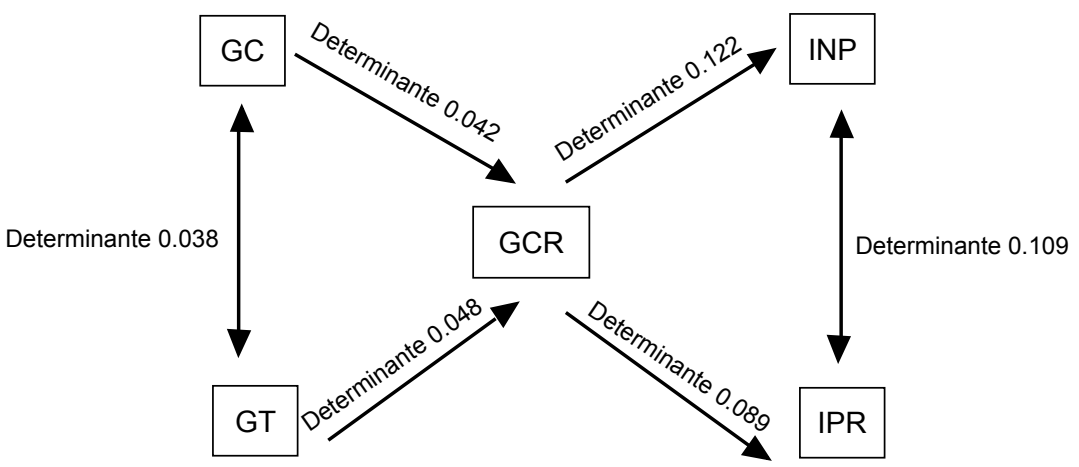

Figura 3. Sistema integrado de gestión de la innovación (SIGI) Elaboración propia

Se puede concluir que en un sistema integrado de gestión de la innovación propuesto, como se muestra en la figura 3, la gestión del conocimiento (GC) y la gestión de la tecnología (GT) influyen positivamente en la gestión de la creatividad (GCR) y que la gestión de la creatividad (GCR) influye positivamente en la innovación de productos (INP) y en la innovación de procesos (IPR). Además, existe una influencia positiva entre la gestión del conocimiento (GC) y la gestión de la tecnología (GT), y entre la innovación de productos (INP) y la innovación de procesos (IPR).

\section{RECOMENDACIONES}

La primera recomendación de la presente investigación radica en que si bien solo se analizó un sector de la industria del país, el sector agroindustrial, sin embargo es replicable a otros sectores, puesto que la gestión del conocimiento (GC), la gestión de la creatividad (GCR), la gestión de la tecnología (GT), la innovación en productos (INP) y la innovación en procesos (IPR) es de aplicación en las empresas, independientemente del sector en el cual se encuentren.

El número de encuestas realizadas también es otro factor que se debe mejorar en futuras investigaciones; se encuestaron 62 empresas del sector agroindustrial, de un universo que se había ubicado en aproxi- 
madamente 120 empresas, por lo que se debería ampliar el número de encuestas para confirmar los resultados obtenidos hasta la fecha, en otros sectores manufactureros del país.

En futuras investigaciones se deberá establecer o proponer la forma como debemos calcular o medir esos medios de control establecidos en el presente artículo, en forma de indicadores, para controlar la gestión de la innovación en las empresas como un sistema estructurado y continuo. Asimismo, explorar la medida en que su organización es nominalmente innovadora o si la innovación está integrada en toda la organización, e identificar las áreas de mejora (Adams, Bessant y Phelps, 2006).

Falta gestión de la innovación en las empresas en forma sistemática y estructurada, lo que limita en alguna medida que se establecezca la situación de la gestión de la innovación en estas, por lo que se deberá proponer una metodología que haga un diagnóstico al respecto, a partir de los medios de control propuestos, así como el planteamiento de un sistema integrado de gestión de la innovación (SIGI) para generar, en forma permanente, sus ventajas o fortalezas, y una mayor productividad vía una gestión de innovación.

Finalmente, se debe hacer un análisis de ecuaciones estructurales que complemente el análisis factorial realizado para reafirmar la relación entre los medios de control de la gestión de la innovación y la estructura del sistema integrado de dicha gestión, que se propone en la presente investigación.

\section{REFERENCIAS}

Adams, R., Bessant, J., y Phelps, R. (2006). Innovation management measurement: A review. International Journal of Management, $8(1), 21-47$.

Arévalo-Tomé, R., Urgal, B., y Quintás, M. (2011). Propuesta de medida del desempeño innovador: aplicación en las empresas innovadoras españolas. Cuadernos de Gestión, 13(1), 41-68.

Ayhan, M., Oztemel, E., Aydin, M., y Yue, Y. (2013). A quantitative approach for measuring process innovation: a case study in a manufacturing company. Revista Internacional de Producción, 51(11), 3463-3475. 
Bain, P., Mann, L., y Pirola-Merlo, A. (2001). The innovation imperative - the relationships between team climate, innovation and performance in research and development teams. Small Group Research, 32(1), 55-73.

Bardales Saucedo, E. (13 de noviembre de 2014). Agroexportaciones de Perú superarán a las de Chile. Gestión. Recuperado de http://gestion.pe/economia/agroexportaciones-peru-superaranchile-2113760

Bhatnagar, J. (2014). Mediator analysis in the management of innovation in Indian knowledge workers: the role of perceived supervisor support, psychological contract, reward and recognition and turn over intention. International Journal of Human Resource Management, 25(10), 1395-1416.

Birkinshaw, J., Hamel, G., y Mol, M. (2008). Management innovation. Academy of Management Review, 33(4), 825-845.

Damanpour, F., y Aravind, D. (2012). Managerial innovation: conceptions, processes and antecedents. Management and Organization Review, 8(2), 423-454.

De Prato, G., y Nepelski, D. (2013). A framework for assessing innovation collaboration partners and its application to BRICs. Revista Internacional de Tecnología de Gestión, 62(2-4), 102-127.

Epsstein, R., Kaminaka, K., Phan, V., y Uda, R. (2013). How is creativity best management? Some empirical and theoretical. Gestión de la Creatividad e Innovación, 22(4), 359-374.

Frishammar, J., Kurkkio, M., Abrahamsson, L., y Lichtenthaler, U. (2012). Antecedents and consequences of firms, process innovation capability: a literature review and a conceptual frameworks. IEEE Transactions on Engineering Management, 59(4), 519-529.

González-Mieres, C., Santos-Vijande, M., y López-Sánchez, J. (2013). An assessment of innovativeness in KIBS: implications on KIBS co-creation culture innovation capability and performance. Revista de Negocios y Marketing Industrial, 28(1-2), 86-101.

Hacklin, F., y Wallin, M. (2013). Convergence and interdisciplinarity in innovation management: a review, critique, and future directions. Service Industries Journal, 33(7-8), 774-788. 
Hagedoorn, J., y Cloodt, M. (2003). Medir el desempeño innovador: ¿existe una ventaja en el uso de múltiples indicadores? Research Policy, 32(8), 1365.

Hollen, R. M., Van den Bosch, F. A., y Volberda, H. W. (2012). The role of management innovation in enabling technological process innovation: an interorganizational perspective. European Management, 10(1), 35-50.

Luna, H.-S. (2013). The relative efficiency analysis of innovation activities with uncertainty: The case of Korean electronic equipment industry. Politica de Innovación para la Gestión y Práctica, 3(15), 305-314.

Molina-Castillo, F.-J., y Munuera-Alemán, J.-L. (2009). New products performance indicators: time horizon and importance attributed by managers. Technovation, 714-724.

Perdomo-Ortiz, J., González-Benito, J., y Galende, J. (2009). An analysis of the relationship between total quality managementbased human resource management practices and innovation. International Journal of Human Resource, 20(5), 1191-1218.

Pohlmann, M., Gebhardt, C., y Etzkowitz, H. (2005). The development of innovation systems and the art of innovation management - strategy, control and the culture of innovation. Technology Analysis \& Strategic, 17(1), 1-7.

Poskela, J., y Martinsuo, M. (2009). Management control and strategic renewal in the front end of innovation. Revista de Productos de Gestión de la Innovación, 26(6), 671-684.

Prajogo, D., y Ahmed, P. (2006). Las relaciones entre estímulos de innovación, capacidad de innovación y resultados de innovación. $R \&$ D Management, 36(5), 499-515.

Quintane, E., Casselman, M., Sebastian, R., y Nylund, P. (2011). Innovation as a knowledge-based outcome. Journal of Knowledge Management, 15(6), 928-947.

Siguaw, J. A., Simpson, P. M., y Enz, C. A. (2006). Conceptualizing innovation orientation: a framework for study and integration of innovation research. Journal of Product Innovation Management, 23(6), 556-574. 
Urgal, B., Quintás, M., y Arévalo-Tomé, R. (2013). Knowledge resources and innovation performance: the mediation of innovation capability moderated by management commitment. Tecnologia de Análisis y Gestión Estratégica, 25(5), 543-565.

Vangelis, S. (2002). Competencias específicas de las empresas que determinan la innovación tecnológica, una encuesta en Grecia. $R \&$ D Management, 32(1), 61. 
